\title{
Mobility and cognition: End points for dietary interventions in aging
}

\author{
Marshall G. Miller ${ }^{\mathrm{a}, \mathrm{b}}$, Derek A. Hamilton ${ }^{\mathrm{c}}$, James A. Joseph ${ }^{\mathrm{a}}$ and Barbara Shukitt-Hale ${ }^{\mathrm{b}, *}$ \\ ${ }^{\mathrm{a}}$ Tufts University, Medford, MA, USA \\ ${ }^{\mathrm{b}}$ United States Department of Agriculture - Agricultural Research Services, Human Nutrition Research Center on \\ Aging, Tufts University, Boston, MA, USA \\ ${ }^{\mathrm{c}}$ University of New Mexico, Albuquerque, NM, USA
}

\begin{abstract}
.
BACKGROUND: Healthy aging is associated with functional declines in mobility and cognition among both humans and non-human animals.

OBJECTIVE: This study combines human measures of mobility and cognition to develop a test battery for evaluating the effects of dietary supplements among older adults. Selected measures parallel behavior tasks used to assess the efficacy of dietary interventions in rodent models of aging.

METHODS: Seventy six healthy adults, between the ages of 21 and 75, completed a 1 hour mobility and cognition assessment. Postural sway and spatiotemporal gait parameters were measured using a Zebris ${ }^{\mathrm{TM}}$ instrumented treadmill system. A computerbased virtual water maze and the trail-making test were used to assess spatial memory and executive function, respectively. RESULTS: Sway velocity during quiet standing increased with age. Preferred gait speed declined with age and changes in the gait cycle reveal an age-related increase in total double support during normal walking. In the virtual water maze, latency to locate a hidden or visible platform increased with age; meanwhile, probe test performance declined with age.

CONCLUSIONS: Healthy adults show age-related declines in measures of mobility and cognition. These measures are a useful tool for translating research on dietary interventions to older adults.
\end{abstract}

Keywords: Aging, postural balance, gait, mobility limitation, cognition, executive function, learning, maze learning, diet therapy

Population aging in developed countries has highlighted the health concerns of older adults. A variety of pathologies is associated with the aging process, including cardiovascular disease, cancer, cataract, dementia, hypertension, metabolic disorder, and osteoporosis. However, 'normal' or healthy aging is also associated with behavioral impairments including functional declines in both mobility and cognition. Significantly, these age-related declines occur even in the absence of degenerative diseases such as Alzheimer's or Parkinson's disease. In combination, these agerelated effects contribute to increased need for assisted living and cost of healthcare among older adults.

${ }^{*}$ Corresponding author: Barbara Shukitt-Hale, Neuroscience and Aging Laboratory, USDA HNRCA at Tufts University, 711 Washington Street, Boston, MA 02111, USA. Tel.: +1 617 556 3118; Fax: +1 617556 3299; E-mail: barbara.shukitthale@ars.usda.gov.
During aging, muscle strength and range of motion decline and movements are slower and more variable [1]. These musculoskeletal alterations, combined with age-related declines in the proprioceptive, cutaneous, visual, and vestibular systems, contribute to impaired postural control, increased fall risk, and inactivity [2]. Postural sway, the constant displacement and correction of the center of gravity within the base of support, is a frequently used aggregate measure of postural control and fall risk. Postural sway during quiet standing increases with age [3] and is predictive of retroactive and prospective falls among older adults $[4,5]$, particularly indoor falls.

Similarly, gait also undergoes changes during aging. With increased age, walking speed declines [6] and recent research has shown that gait speed, in particular, is positively associated with life expectancy in a pooled 
analysis of 34,485 older adults from nine separate cohorts [7]. Aging is also associated with increases in the variability of spatial and temporal gait parameters, such as step width, stride length, stride time, percent double support time (both feet on ground); which have each been associated, retrospectively or prospectively, with risk of falls [8-12]. Increases in step-width variability have been observed during aging, even among non-fallers [13-15]. Medial-lateral instability due to increased step-width variability during gait may be particularly problematic for older adults as falls to the side, rather than to the front or back, are associated with a three-fold increase in risk of hip fracture [16]. While increases in gait variability have been the focus of much research, there is also evidence that decreased gait variability may also signal an increased risk of falls and functional decline [11], perhaps due to inflexibility in response to obstacles or perturbation.

Cognition, like balance and gait, undergoes changes during the aging process. While some aspects of cognition, such as knowledge, appear to improve with age, other aspects such as processing speed, reasoning, and memory decline with age [17]. Declines have also been observed in spatial navigation and spatial aspects of learning and memory during aging [18, 19], which may contribute to spatial disorientation among older adults. Declining executive function, attention, and ability to divide attention between multiple tasks may also contribute to risk of falls and decreased productivity [20-23]. In humans, decline in executive function is associated with impaired balance, gait, and spatial navigation [24-27].

Recent research has linked age-related changes in cognition with those in mobility [28-35], although the links between specific cognitive domains and individual gait and balance parameters are not fully understood. Declines in various gait parameters have also been linked directly to age-related atrophy in brain regions critical to various cognitive domains [36].

Similar functional declines are also observed in non-human animals. A study in our laboratory [37] found significant, age-related decline across a variety of behavioral tasks in rats from 6 to 22 months of age. Young rats were able to maintain postural control despite an artificially narrow base of support while aged rats fell significantly sooner. Similarly, young rats were able to maintain their gait atop an accelerating rotarod while aged rats fell off more quickly and at slower speeds. Finally, young rats quickly learned to locate a hidden platform in a working memory version of the Morris water maze (MWM) [38] while aged rats showed significant impairment.

In a recent review, it was argued that a considerable shortcoming of tests used in human intervention studies is that the [cognitive] tests used to evaluate the effects of the intervention bear little resemblance to the tasks used in the rodent literature on which the human trials are based [39]. The present study evaluates postural sway, gait, spatial navigation, and executive function in a sample of adults between the ages of 21 and 75 years of age. The aim of the study is to examine age-sensitive human measures of mobility and cognition that reflect the age-related changes observed in our rodent model of aging. It was hypothesized that performance on these tasks would decrease significantly across age-groups, replicating prior findings in older adults and rodent models of aging.

\section{Methods}

\subsection{Participants}

Men and women were recruited into one of six groups according to their age (21-40, 41-50, 51-60, $61-65,66-70$, and 71-75 years of age). Age ranges were selected to aid in identifying an age range during which participants demonstrated measureable performance declines but were still able to safely complete all of the measures. Inclusion criteria included: fluency in written and spoken English, normal (20/20) or corrected to normal vision, and body mass index (BMI) between 18.5 and 30. Exclusion criteria included: recent hip or lower-limb surgery, recent head or lower body injury, use of prosthetics, established psychological or neurological disorders, liver or kidney disease, or the use of medicines or supplements that may have affected study outcomes. Participants were compensated $\$ 10$. This study was approved by the Tufts medical institutional review board. Written informed consent was obtained from all study participants.

\subsection{Stance analysis}

Stance analysis was performed using an instrumented treadmill (Zebris ${ }^{\mathrm{TM}}$ ). During each 1-minute stance assessment, a high-density array of pressure sensors beneath the tread belt recorded pressure variation. Participants were instructed to stand as still and straight as possible and measurements were taken in 
both the eyes-open and eyes-closed condition in counterbalanced order. Stance was assessed before and after gait analysis. The associated software package (WinFDM-T, Zebris ${ }^{\mathrm{TM}}$ ) then computed a variety of postural sway measurements from the center of pressure (COP), which included: length of COP path (velocity) and the area $\left(\mathrm{mm}^{2}\right)$ of the COP $95 \%$ confidence ellipse.

\subsection{Gait analysis}

Gait analysis was also performed using the instrumented treadmill (Zebris ${ }^{\mathrm{TM}}$ ). During gait, pressure sensors detected each footfall and the associated software package (WinFDM-T, Zebris ${ }^{\mathrm{TM}}$ ) then computed a variety of spatial and temporal measures from this data, including: step length, step width, step duration, stride length, stride duration, cadence (strides/min), foot rotation, and percent of gait cycle spent in each gait phase (e.g. load, single support, double support, etc.). Participants were blind to treadmill speed settings.

\subsection{Cognition}

The virtual Morris water maze (vMWM; Neuroinvestigations, Inc) is a computerized version of the standard Morris water maze typically employed to test spatial navigation, hippocampal function, and working/reference memory in rodents [38]. In this task, participants used a keyboard to navigate a threedimensional (3D) virtual environment containing a circular pool surrounded by multiple distal cues to locate a goal platform. After receiving instructions, participants completed two training trials to familiarize themselves with the software. Participants then completed a series of 16 acquisition trials wherein they explored the pool to locate an invisible platform. Participants began each acquisition trial in a pseudorandom starting location (without repetition) and the trial ended when the participant moved into the platform's location. If, on any of the trials, a participant failed to reach the invisible platform within 60 seconds, the platform became visible and the participant was instructed to move directly to it. Participants then completed a 40 second probe test, which was identical to the previous learning trials except that moving through the platform's location did not end the trial. Participants also completed four trials in which the platform was visible throughout the whole trial. Each trial was separated by a 2 second inter-trial interval.
Psychomotor speed and executive function were assessed using the trail making test (TMT) [40]. The TMT is a two-part paper-and-pencil assessment wherein participants sequentially connect a series of numbered and/or lettered circles on a sheet of paper. Participants completed Part A by drawing a continuous line connecting circles numbered 1 through 25 . Participants then completed Part B by drawing a continuous line connecting circles numbered 1 through 13 and circles lettered "A" through "L." Numbers and letters were connected in alternating order (e.g. A, 1, B, 2, C, 3...). If participants made sequence errors during either part, they were asked to start again from the last correctly connected circle. Latency to connect the circles during each part was recorded using a stopwatch.

\subsection{Procedure}

On the test day, participants were consented and began by completing the first stance assessment. Then, the participants were habituated to walking on the treadmill at $1.5 \mathrm{mph}$ for 6 minutes. The treadmill speed was then increased and decreased three times and participants were allowed to self-select their preferred walking speed. Participants walked at their preferred walking speed for 4 minutes while their gait was recorded. Participants then repeated the stance assessment procedure after walking on the treadmill. Participants then completed the vMWM, TMT, and demographic, activity, and fall history questionnaires.

\subsection{Analysis}

Systat (Systat Software, Inc.) was used to conduct all statistical analyses. Seventy-eight participants were recruited; however, four participants were dropped from the analysis due to significant overweight or inability to complete all study tasks (see Table 1 for participant demographics). Outliers, defined as results $>2$ standard deviations from the mean, were removed from the analysis. For analysis, participants in the six age-groups $(21-40,41-50,51-60,61-65,66-70$, or 71-75 years of age) were compared using analysis of variance (ANOVA). Post hoc analyses were conducted on all significant results using Fisher's LSD. Analysis of covariance was also conducted for all significant results. Alpha levels were assessed at the 0.05 level. 
Table 1

Participant demographics presented as mean \pm standard deviation

\begin{tabular}{|c|c|c|c|c|c|c|}
\hline \multirow[t]{2}{*}{ Demographics } & \multicolumn{6}{|c|}{ Age (years) } \\
\hline & $21-40$ & $41-50$ & $51-60$ & $61-65$ & $66-70$ & $71-75$ \\
\hline$n$ & 13 & 12 & 13 & 12 & 12 & 12 \\
\hline Female & $54 \%$ & $50 \%$ & $54 \%$ & $50 \%$ & $50 \%$ & $50 \%$ \\
\hline Age (y) & $28.8 \pm 5.1$ & $45.5 \pm 3.0$ & $56.2 \pm 3.0$ & $62.8 \pm 1.5$ & $68.3 \pm 1.5$ & $73.3 \pm 1.3$ \\
\hline Height(m) & $1.7 \pm 0.1$ & $1.7 \pm 0.1$ & $1.7 \pm 0.1$ & $1.8 \pm 0.1$ & $1.7 \pm 0.1$ & $1.8 \pm 0.1$ \\
\hline Weight(kg) & $76.4 \pm 16.7$ & $67.5 \pm 14.3$ & $70.7 \pm 13.2$ & $81.8 \pm 14.6$ & $74.4 \pm 15.5$ & $76.0 \pm 14.2$ \\
\hline BMI $\left(\mathrm{kg} / \mathrm{m}^{2}\right)$ & $25.3 \pm 6.0$ & $22.7 \pm 4.6$ & $24.8 \pm 3.9$ & $26.4 \pm 3.0$ & $26.6 \pm 5.1$ & $24.9 \pm 4.7$ \\
\hline Education (y) & $16.1 \pm 3.5$ & $17.1 \pm 3.9$ & $16.1 \pm 5.7$ & $18.0 \pm 3.6$ & $15.0 \pm 3.3$ & $14.5 \pm 2.3$ \\
\hline Falls in past year & $15 \%$ & $25 \%$ & $38 \%$ & $17 \%$ & $8 \%$ & $25 \%$ \\
\hline
\end{tabular}

\section{Results}

\subsection{Stance}

Stance data were analyzed using a six age-group by two time-point (before or after gait assessment) by two eye-condition (eyes open, eyes closed) mixed model analysis of variance (ANOVA). Analysis revealed an effect of age-group on sway velocity $[F(5,66)=5.548, p<0.001]$, an effect of time $[F(1$, $66)=5.361, p=0.024]$, and an effect of eye condition $[F(1,66)=242.976, p<0.001]$ (Fig. 1). Post hoc analysis shows that, relative to the youngest age-group, sway velocity was increased in age-groups 51 years and older in the eyes open condition and in age-groups 61 years and older in the eyes closed. For all age groups, sway velocity was increased in the eyes closed condition. Sway velocity was slightly lower during the second assessment $[F(1,66)=5.361, p=0.024]$ and the effect of the eyes closed condition was less pronounced during the second measurement. A significant interaction was also observed between age-group and eye condition $[F(5,66)=5.336, p<0.001]$ (Fig. 1), with sway velocity increased more in the eyes closed condition among older age groups. When gender, height and weight were controlled, only an effect of age-group $(p<0.001)$ and an age-group by eye condition interaction $(p=0.001)$ remained significant. Height was a significant covariate $(p=0.041)$, indicating that taller participants also had increased COP path lengths.

Analysis revealed no effect of age on sway area ( $p=0.232$; data not shown). However, sway area was increased during the second measurement $[F(1,66)=$ $4.080, p=0.047]$, and sway areas were increased in the eyes closed condition $[F(1,66)=10.301, p=0.002]$, in all age-groups. When gender, height, and weight were controlled, no significant effects remained.

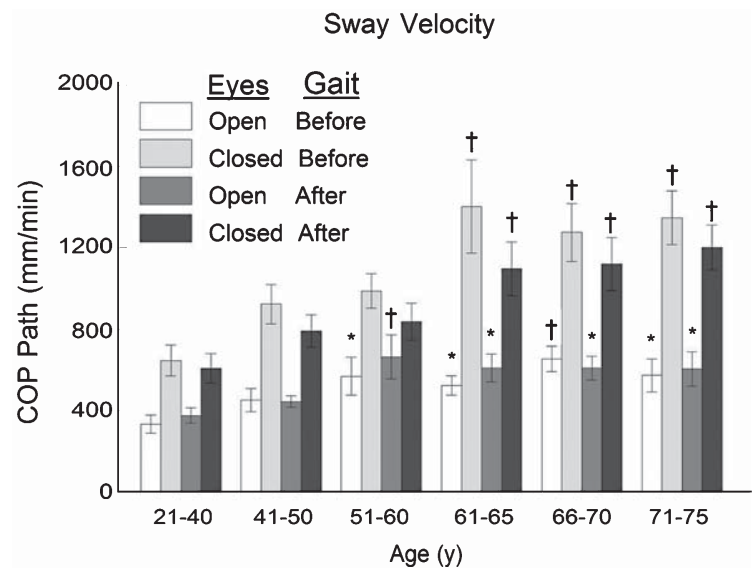

Fig. 1. Sway velocity presented as $\mathrm{mm} /$ minute change in center of pressure (COP). Error bars denote SEM. Asterisks and crosses indicate a significant difference from the youngest age-group (21-40 yo): ${ }^{*}=p<0.05 ;{ }^{\dagger}=p<0.01$.

\subsection{Gait}

All gait measures were collected while participants walked at their preferred walking speed and analyzed using a six age-group ANOVA. Analysis revealed an effect of age-group on preferred walking speed $[F(5$, $68)=2.700, p=0.028$ ], which remained significant even when gender, height, and weight were controlled ( $p=0.037$; see Table 2). Post hoc analysis shows that the 51-60, 66-70, and 71-75 age-groups had significantly lower preferred walking speeds than did the 21-40 year old group. An effect of age-group on stride length was also observed $[F(5,68)=4.465, p=0.001]$, which also remained significant when gender, height, and weight were controlled ( $p=0.011$; Table 2). Post hoc analysis shows that the 41-50, 51-60, 66-70, and 71-75 age-groups had significantly shorter stride 
Table 2

Gait measures and cycle presented as mean \pm standard deviation

\begin{tabular}{|c|c|c|c|c|c|c|}
\hline \multirow[t]{2}{*}{ Gait } & \multicolumn{6}{|c|}{ Age (years) } \\
\hline & $21-40$ & $41-50$ & $51-60$ & $61-65$ & $66-70$ & $71-75$ \\
\hline Preferred Speed $(\mathrm{km} / \mathrm{h})$ & $6.4 \pm 1.1$ & $5.6 \pm 0.8$ & $5.3 \pm 1.4^{*}$ & $6.1 \pm 1.4$ & $5.3 \pm 1.0^{*}$ & $5.1 \pm 1.3^{*}$ \\
\hline Stride Length (cm) & $128.4 \pm 18.7$ & $109.4 \pm 12.2^{\dagger}$ & $103.7 \pm 16.3^{\dagger}$ & $119.2 \pm 21.6$ & $103.8 \pm 16.2^{\dagger}$ & $106.5 \pm 13.7^{\dagger}$ \\
\hline \multicolumn{7}{|l|}{ Gait Cycle } \\
\hline Stance Phase $(\%)$ & $63.0 \pm 2.9$ & $63.8 \pm 2.6$ & $65 . \pm 2.6^{\dagger}$ & $63.8 \pm 2.6$ & $66.1 \pm 2.0^{\dagger}$ & $65.8 \pm 1.6^{\dagger}$ \\
\hline Load $(\%)$ & $13.0 \pm 2.9$ & $13.7 \pm 2.8$ & $15.7 \pm 2.6^{\dagger}$ & $13.8 \pm 2.6$ & $16.2 \pm 2.0^{\dagger}$ & $15.8 \pm 1.6^{\dagger}$ \\
\hline Single $(\%)$ & $37.0 \pm 2.9$ & $36.3 \pm 2.8$ & $34.3 \pm 2.6^{\dagger}$ & $36.2 \pm 2.6$ & $33.8 \pm 2.0^{\dagger}$ & $34.2 \pm 1.6^{\dagger}$ \\
\hline Pre-Swing (\%) & $13.0 \pm 2.9$ & $13.8 \pm 2.6$ & $15.7 \pm 2.6^{\dagger}$ & $13.8 \pm 2.6$ & $16.2 \pm 2.0^{\dagger}$ & $15.8 \pm 1.6^{\dagger}$ \\
\hline Swing Phase (\%) & $37.0 \pm 2.9$ & $36.2 \pm 2.6$ & $34.3 \pm 2.6^{\dagger}$ & $36.2 \pm 2.6$ & $33.8 \pm 2.0^{\dagger}$ & $34.2 \pm 1.6^{\dagger}$ \\
\hline Double Support (\%) & $25.9 \pm 5.8$ & $27.4 \pm 5.4$ & $31.4 \pm 5.2^{\dagger}$ & $27.5 \pm 5.2$ & $32.4 \pm 4.0^{\dagger}$ & $31.7 \pm 3.2^{\dagger}$ \\
\hline
\end{tabular}

Asterisks and crosses indicate a significant difference from the youngest age-group $(21-40$ yo $):^{*}=p<0.05 ;{ }^{\dagger}=p<0.01$.

lengths than did the $21-40$ year old group. The covariate, height, was significantly related to stride length $(p=0.006)$, wherein taller participants took longer strides. To quantify gait variability, coefficients of variation (CV; SD/mean) were calculated for step width, stride length, and stride time; however, no significant differences were observed in step width $\mathrm{CV}$, stride length $\mathrm{CV}$, or stride time $\mathrm{CV}$, nor were differences observed in step width or stride time.

Gait cycle measures were averaged between left and right legs for each participant. All phases of the gait cycle varied significantly by age-group (Table 2 ). An effect of age-group on load, pre-swing, and total double support phases was observed $[F \mathrm{~s}(5,68)=3.809$, $p \mathrm{~s}=0.004]$ and was significant when gender, height, and weight were controlled $(p s=0.002)$. Post hoc analysis shows that these phases represent a larger percent of the gait cycle among participants in the 51-60, 66-70, and 71-75 year old age-groups, relative to 21-40 year old participants. The covariate, weight, was significantly related to all three phases ( $p \mathrm{~s}=0.003,0.002$, and 0.002 , respectively), wherein heavier participants spent more time in these phases. An effect of age-group on single support and swing phases was also observed $[F \mathrm{~s}(5,68)=3.805$ and 3.809 , respectively, $p s=0.004]$, which also remained significant when gender, height, and weight were controlled ( $p$ s $=0.002$ ). Post hoc analysis shows that these phases represent a smaller percent of the gait cycle among participants in the 51-60, 66-70, and 71-75 year old age-groups, relative to 21-40 year old participants. The covariate, weight, was significantly related to both phases ( $p s=0.003$ and 0.002 , respectively), wherein heavier participants spent less time in these phases.

\subsection{Cognition}

Acquisition trials in the vMWM were averaged into four blocks of four trials and data were analyzed using a six age-group by four block mixed model ANOVA. An effect of age-group on latency to locate the hidden platform was observed $[F(5,65)=8.311, p<0.001]$ as well as an effect of acquisition block $[F(3,195)=4.946$, $p=0.002]$ and an age-group by acquisition block interaction $[F(15,195)=1.832, p=0.033]$ (Fig. 2A). While participants in all age-groups located the hidden platform significantly faster from block to block during acquisition, participants in the 21-40 year old agegroup showed larger decreases in latencies than did those in older age groups. Analysis also revealed a significant interaction between acquisition blocks and the covariate, years of education $[F(3,194)=3.060$, $p=0.029$ ], indicating a relationship between higher education and speed of acquisition. Latency during acquisition trials was also summed and analyzed using a six group ANOVA. Analysis revealed an effect of age-group on summed acquisition latencies $[F(5$, $65)=8.311, p<0.001]$ (Fig. 2B). Post hoc analysis shows that all of the other age-groups had significantly higher summed latencies than the 21-40 year old group. Visible platform trials in vMWM were averaged into a single block of four trials and analyzed using a six age-group ANOVA. An effect of age-group on latency to reach the visible platform was also observed $[F(5,65)=3.647, p=0.006]$ (Fig. 2E). Post hoc analysis shows that the 66-70 and 71-75 year old age-group took significantly longer than the $21-40$ year old group.

Probe trial measures were analyzed using a six age-group ANOVA. An effect of age-group on the percent of time spent in the trained quadrant was 

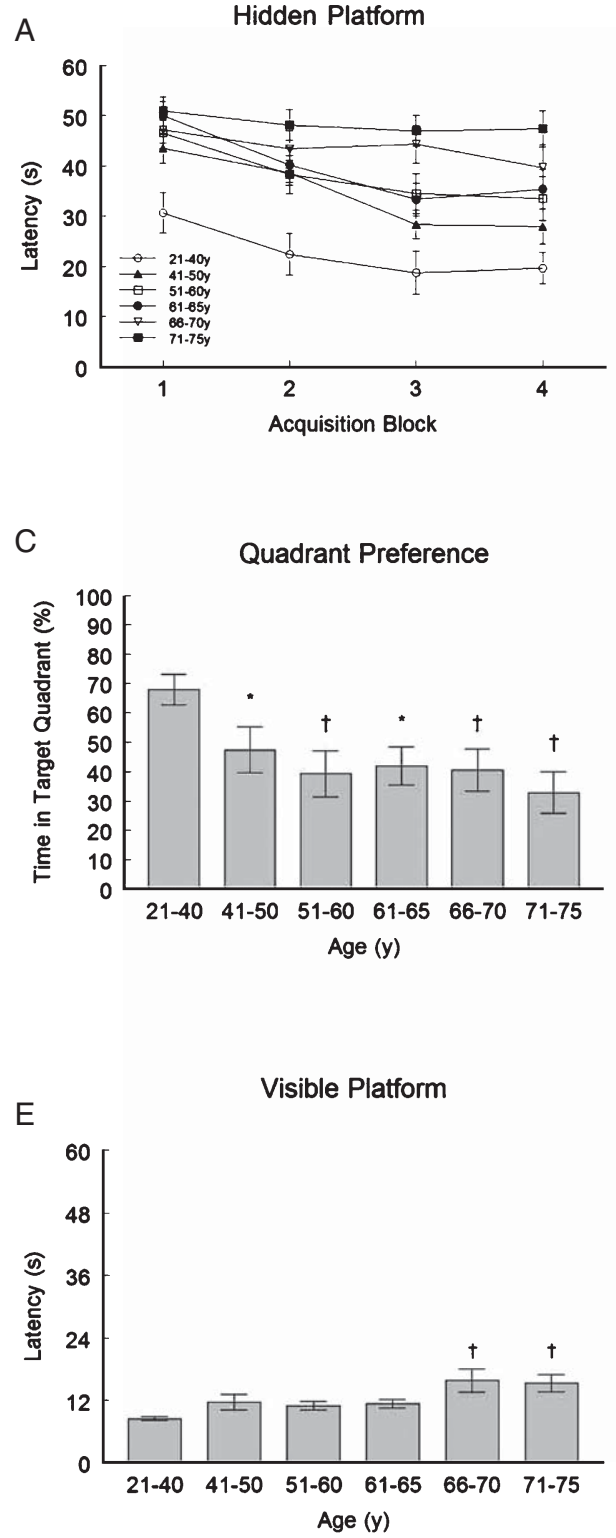
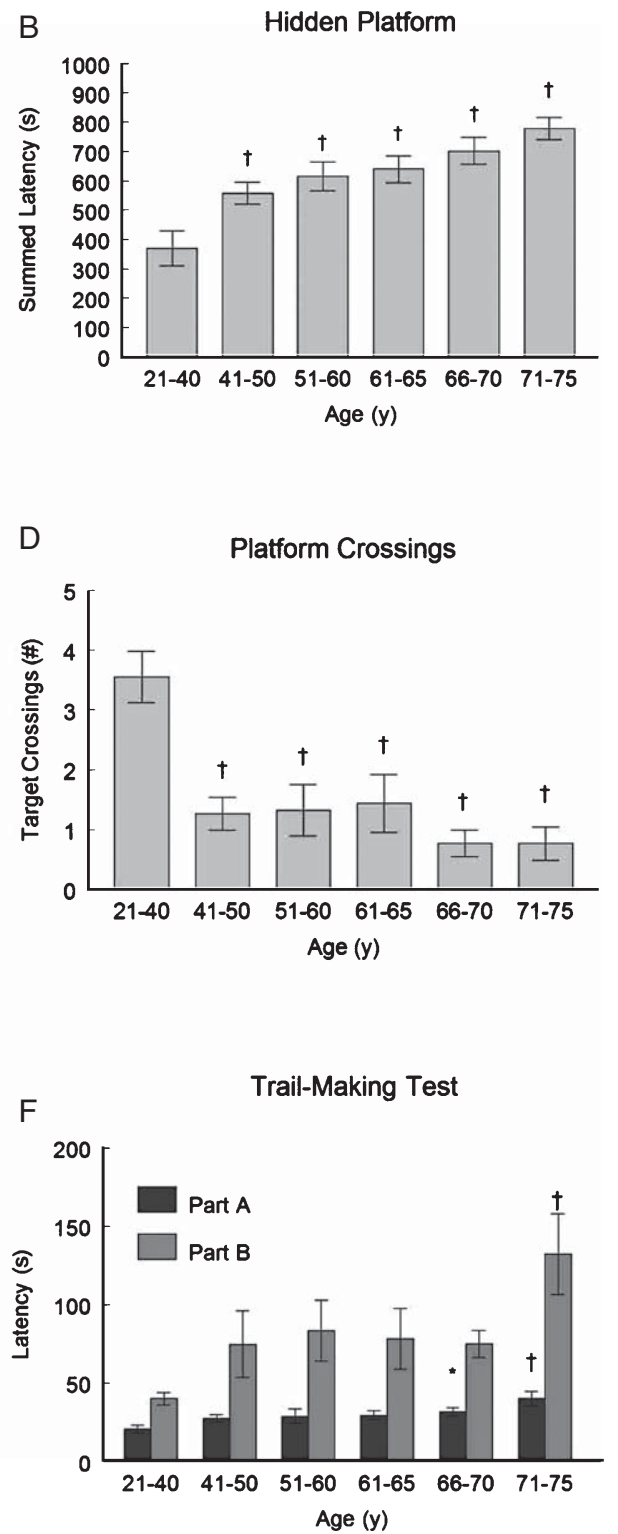

Fig. 2. A) Virtual Morris water maze (vMWM) acquisition presented as latency to locate a hidden platform during 4 blocks of 4 trials. B) vMWM acquisition presented as summed latency to locate a hidden platform during all 16 acquisition trials. C) vMWM probe test performance presented as percent of time spent searching in the goal quadrant. D) vMWM probe test performance presented as number of platform location crossings. E) vMWM control trials presented as latency to reach a visible platform. F) Trail-making-test (TMT) performance presented as latency to complete Part A and Part B. All error bars denote SEM. Asterisks and crosses indicate a significant difference from the youngest age-group $\left(21-40\right.$ yo): ${ }^{*}=p<0.05 ;{ }^{\dagger}=p<0.01$.

observed $[F(5,65)=2.758, p=0.025]$ (Fig. 2C). Analysis showed a trend toward an effect of age-group on increased time spent in the opposite quadrant with increased age $[F(5,68)=2.065, p=0.080]$. Similarly, analysis showed a trend toward an effect of age-group on increased mean proximity to the platform's location
$[F(5,68)=2.039, p=0.085]$, indicating that younger participants searched closer to the platform's previous location during the probe trial, relative to older participants (data not shown). An effect of age-group on platform crossings was observed $[F(5,65)=7.806$, $p<0.001$ ] (Fig. 2D). Post hoc analysis shows that all 
of the other age-groups spent significantly less time in the trained quadrant and crossed the platform's location fewer times, relative to the 21-40 year old group.

Performance on the TMT was analyzed using a six age-group ANOVA. Analysis revealed an effect of age-group on latency to complete Part A $[F(5$, $65)=2.572, p=0.035]$ (Fig. 2F). Post hoc analysis shows that the 66-70 and 71-75 year old age-groups took significantly longer than the 21-40 year old group. While a significant effect of age-group on latency to complete Part B was observed $[F(5,68)=2.759$, $p=0.025]$, the effect was abolished $(p=0.109)$ when gender $(p=0.668)$ and years of education $(p=0.162)$ were included as covariates. Post hoc analysis shows that only the 71-75 year old age-group took significantly longer to complete Part B, relative to the 21-40 year old group.

\section{Discussion}

This study reports 3 main findings: 1) postural control during quiet standing declines with age; 2) preferred walking speed declines with age; and 3) spatial working memory declines with age. Furthermore, results on each measure parallel those observed in rodent models of aging and replicated prior findings in aging humans.

In the present study, adults from each age-group stood quietly atop a pressure sensor array while sway velocity was measured in both the eyes open and eyes closed condition. Age had a significant effect on sway velocity as did eye-condition (eyes open vs closed), particularly among participants in the 61-65, 66-70, and 71-75 age-groups. Other studies have also reported increased postural sway with age, even in the absence of chronic disease or injury [3, 41, 42]. Age-related changes in postural control negatively impact older adults and often presage a transition from independent to assisted living. Postural instability is a key risk factor for the increased incidence of falls among older adults [43], which often result in injury and an increased risk of subsequent falls. Even if no injury occurs, falls often lead to increased fear of falling, inactivity, and social withdrawal [44-46], which also contribute to the risk of subsequent falls. Increased postural sway during quiet standing among older adults is an important factor in predicting falling, even without recent fall history or apparent balance impairment $[4,5,47]$.
Participants also walked on an instrumented treadmill while spatiotemporal parameters of their gait were recorded. Participants in older age-groups preferred slower walking speeds and demonstrated decreased stride lengths relative to participants in the 21-40 year old age-group. These results support those of previous studies that report a slowing of gait with age $[6,48-51]$. Results from this study also show that the gait cycle is altered during aging. Interestingly, participants in the 61-65 year old age-group demonstrated gait patterns that were inconsistent with the overall trend; however, this may be an artifact of recruitment wherein these participants necessarily were able to walk to jobs in an urban city-center. Prior studies have also reported an increase in double support with age $[48,49]$ and it has been suggested that this increase may be a gait strategy to promote stability. The observed age-related changes in gait parameters are also consistent with alterations associated with fear of falling [52, 53]. No significant differences in step width, stride length, or stride time variability were detected in this sample; however, nonsignificant changes were in the expected direction (data not shown).

The Morris water maze (MWM) [38] has become a gold standard for assessing spatial ability in rodents [19], which is sensitive to hippocampal [54] and striatal dysfunction [55]. Humans perform similarly to rodents when tested in virtual versions of the MWM [56-58] where performance is also dependent on hippocampal and striatal integrity [59-61]. In the present study, young adults (21-40 years old) performed significantly better than adults in the other age-groups during acquisition as well as on the probe test. Latency to locate a visible platform increased slightly in the 66-70 and 71-75 year old age-groups, perhaps reflecting generational differences in computer familiarity or an age-related decline in psychomotor speed, as seen in the TMT (see below). However, the effect of age on other vMWM performance measures was still observed, even when latency to complete visible trials was included as a covariate. These results replicate prior studies that also found age-related performance declines in vMWM performance [18, 19, 62, 63].

The trail making test [40] is a commonly used test of visual search, psychomotor speed, and executive function. Prior studies have reported age-related changes in TMT performance on both Parts A and B [64-66]. In the present study, latency to complete Part A was increased among the 66-70 and 71-75 year old age-groups, suggesting a decline in visual 
search and psychomotor speed. Latency to complete Part B shows a non-significant increase in latency in the 41-50, 51-60, 61-65, and 66-70 year old age-groups and a significant increase in latency in the 71-75 year old age-group. The addition of the covariate years of education, but not gender, attenuated the effect of age in this analysis. These data are consistent with the age norms reported by Tombaugh [66] that also show a performance plateau during middle years among adults with $12+$ years of education but no effect of gender on performance.

Research from our laboratory shows that rodents undergo similar age-related motor and cognitive declines [37] as were seen in the present study. However, dietary interventions with polyphenol-rich whole foods can mitigate and, in some cases, even reverse the age-related performance declines observed in aged animals. Supplementing aged 19 mo rats' diet with blueberry or strawberry improved postural control, gait, and spatial working memory relative to controls [67]. Subsequent studies using these measures have shown that a variety of phenol-rich whole foods can retard or even reverse the effects of age [68-72]. However, while epidemiological studies show that increased fruit and nut intakes are associated with improved mobility and cognition during aging [73], few clinical studies have investigated the effects of individual polyphenol-rich whole foods on mobility and cognition among older adults. We plan to use the tests validated here in subsequent studies to investigate the effects of polyphenolic-rich foods for their ability to improve age-related declines in motor and cognitive performance.

In the present study, tests were chosen to parallel age-sensitive rodent tests which have also proven sensitive to dietary manipulations. Quiet standing with a narrow base of support and treadmill walking are analogous to rodent mobility tasks such as balancing on a narrow plank or rotarod tasks. Similarly, the vMWM is directly modeled on the rodent task. The results from the battery of human mobility and cognition tests chosen in this study provided strong evidence that these tests are age-sensitive and can potentially be applied to future studies that aim to assess the effect of dietary interventions among older adults.

\section{References}

[1] Seidler RD, Bernard JA, Burutolu TB, Flinq BW, Gordon MT, Gwin JT, Kwak Y, Lipps DB. Motor control and aging: Links to age-related brain structural, functional, and biochemical effects. Neurosci Biobehav Rev. 2010;34:721-33.

[2] Horak FB. Postural orientation and equilibrium: What do we need to know about neural control of balance to prevent falls? Age Ageing. 2006;35(Suppl 2):ii7-ii11.

[3] Overstall PW, Exton-Smith AN, Imms FJ, Johnson AL. Falls in the elderly related to postural imbalance. $\mathrm{Br}$ Med J. 1977;1:261-4.

[4] Pajala S, Era P, Koskenvuo M, Kaprio J, Törmäkangas T, Rantanen T. Force platform balance measures as predictors of indoor and outdoor falls in community-dwelling women aged 63-76 years. J Gerontol A Biol Sci Med Sci. 2008;63:171-8.

[5] Maki BE, Holliday PJ, Topper AK. A prospective study of postural balance and risk of falling in an ambulatory and independent elderly population. J Gerontol. 1994;49:M72-84.

[6] Prince C, Corriveau H, Hebert R, Winter D. Gait in the elderly. Gait and Posture. 1997;5:128-35.

[7] Studenski S, Perera S, Patel K, Rosano C, Faulkner K, Inzitari M, Brach J, Chandler J, Cawthon P, Connor EB, Nevitt M, Visser M, Kritchevsky S, Badinelli S, Harris T, Newman AB, Cauley J, Ferrucci L, Guralnik J. Gait speed and survival in older adults. JAMA. 2011;305(1):50-8.

[8] Maki BE. Gait changes in older adults: Predictors of falls or indicators of fear. J Am Geriatr Soc. 1997;45:313-20.

[9] Hausdorff JM, Edelberg HK, Mitchell SL, Goldberger AL, Wei JY. Increased gait unsteadiness in community-dwelling elderly fallers. Arch Phys Med Rehabil. 1997;78:278-83.

[10] Hausdorff JM, Rios DA, Edelberg HK. Gait variability and fall risk in community-living older adults: A 1-year prospective study. Arch Phys Med Rehabil 2001;82:1050-6.

[11] Brach JS, Berlin JE, VanSwearingen JM, Newman AB, Studenski SA. Too much or too little step width variability is associated with a fall history in older persons who walk at or near normal gait speed. J Neuroeng Rehabil. 2005;2:21.

[12] Verghese J, Holtzer R, Lipton RB, Wang C. Quantitative gait markers and incident fall risk in older adults. J Gerontol A Biol Sci Med Sci. 2009;64:896-901.

[13] Grabiner PC, Biswas ST, Grabiner MD. Age-related changes in spatial and temporal gait variables. Arch Phys Med Rehabil. 2001;82(1):31-5.

[14] Owings TM, Grabiner MD. Measuring step kinematic variability on an instrumented treadmill: How many steps are enough? J Biomech. 2003;36(8):1215-8.

[15] Owings TM, Grabiner MD. Step width variability, but not step length variability or step time variability, discriminates gait of healthy young and older adults during treadmill locomotion. J Biomech. 2004;37(6):935-8.

[16] Greenspan SL1, Myers ER, Maitland LA, Resnick NM, Hayes WC. Fall severity and bone mineral density as risk factors for hip fracture in ambulatory elderly. JAMA. 1994;271(2):12833.

[17] Salthouse TA. What and when of cognitive aging. Curr Dir Psychol Sci. 2004;13:140-4.

[18] Moffat SD, Resnick SM. Effects of age on virtual environment place navigation and allocentric cognitive mapping. Behav Neurosci. 2002;116(5):851-9.

[19] Driscoll I, Hamilton DA, Yeo RA, Brooks WM, Sutherland RJ. Virtual navigation in humans: The impact of age, sex, and hormones on place learning. Horm Behav. 2005;47(3): 326-35. 
[20] Inzitari M, Balderschi M, Carlo AD, Bari DM, Marchionni N, Scafato E, Farchi G, Inzitari D. Impaired attention predicts motor performance decline in older community-dwellers with normal baseline mobility: Results from the Italian Longitudinal Study on Aging (ILSA). J Gerontol A Biol Sci Med Sci. 2007;62A(8):837-43.

[21] Yogev G, Hausdorff JM, Giladi N. The role of executive function and attention in gait. Mov Disord. 2008;23(3):329-472.

[22] Montero-Odasso M, Bergman H, Phillips NA, Wong CH, Sourial N, Chertkow H. Dual tasking and gait in people with mild cognitive impairment. The effect of working memory. BMC Geriatrics. 2009;9:41.

[23] Holtzer R, Friedman R, Lipton RB, Katz M, Xue X, Verghese $\mathrm{J}$. The relationship between specific cognitive functions and falls in aging. Neuropsychology. 2007;21(5):540-8.

[24] Rabbitt PM, Scott M, Thacker N, Lowe C, Horan M, Pendleton N, Hutchinson D, Jackson A. Balance marks cognitive changes in old age because it reflects global brain atrophy and cerebro-arterial blood-flow. Neuropsychologia. 2006;44(10): 1978-83.

[25] Verghese J, Wang C, Lipton RB, Holtzer R, Xue X. Quantitative gait dysfunction and risk of cognitive decline and dementia. J Neurol Neurosurg Psychiatry. 2007;78(9):929-35.

[26] Vazzana R, Bandinelli S, Lauretani F, Volpato S, Lauretani F, Di Iorio A, Abate M, Corsi AM, Milaneschi Y, Guralnik JM, Ferrucci L. Trail Making Test predicts physical impairment and mortality in older persons. J Am Geriatr Soc. 2010;58(4):71923.

[27] Sanders AE, Holtzer R, Lipton RB, Hall C, Verghese J. Egocentric and exocentric navigation skills in older adults. J Gerontol A Biol Sci Med Sci. 2008;63(12):1356-63.

[28] Verlinden VJ, van der Geest JN, Hofman A, Ikram MA. Cognition and gait show a distinct pattern of association in the general population. Alzheimers Dement. 2013 [Epub ahead of print]

[29] Mielke MM, Roberts RO, Savica R, Cha R, Drubach DI, Christianson T, Pankratz VS, Geda YE, Machulda MM, Ivnik RJ, Knopman DS, Boeve BF, Rocca WA, Petersen RC. Assessing the temporal relationship between cognition and gait: Slow gait predicts cognitive decline in the Mayo Clinic Study of Aging. J Gerontol A Biol Sci Med Sci. 2013;68(8):929-37.

[30] Martin KL, Blizzard L, Wood AG, Srikanth V, Thomson R, Sanders LM, Callisaya ML. Cognitive function, gait, and gait variability in older people: A population-based study. J Gerontol A Biol Sci Med Sci. 2013;68(6):726-32.

[31] Bruce-Keller AJ, Brouillette RM, Tudor-Locke C, Foil HC, Gahan WP, Nye DM, Guillory L, Keller JN. Relationship between cognitive domains, physical performance, and gait in elderly and demented subjects. J Alzheimers Dis. 2012;30(4):899-908.

[32] Duff K, Mold JW, Roberts MM. Walking speed and global cognition: Results from the OKLAHOMA Study. Neuropsychol Dev Cogn B Aging Neuropsychol Cogn. 2008;15(1):31-9.

[33] Atkinson HH, Rosano C, Simonsick EM, Williamson JD, Davis C, Ambrosius WT, Rapp SR, Cesari M, Newman AB, Harris TB, Rubin SM, Yaffe K, Satterfield S, Kritchevsky SB; Health ABC study. Cognitive function, gait speed decline, and comorbidities: The health, aging and body composition study. J Gerontol A Biol Sci Med Sci. 2007;62(8):844-50.
[34] Holtzer R, Verghese J, Xue X, Lipton RB. Cognitive processes related to gait velocity: Results from the Einstein Aging Study. Neuropsychology. 2006;20(2):215-23.

[35] Hausdorff JM, Yogev G, Springer S, Simon ES, Giladi N. Walking is more like catching than tapping: Gait in the elderly as a complex cognitive task. Exp Brain Res. 2005;164(4):541-8.

[36] Callisaya ML, Beare R, Phan TG, Blizzard L, Thrift AG, Chen J, Srikanth VK. Brain structural change and gait decline: A longitudinal population-based study. J Am Geriatr Soc. 2013;61(7):1074-9.

[37] Shukitt-Hale B, Mouzakis G, Joseph JA. Psychomotor and spatial memory performance in aging male Fischer 344 rats. Exp Gerontol. 1998;33:615-24.

[38] Morris RGM. Spatial localization does not require the presence of local cues. Learning and Motivation. 1981;12:239-60.

[39] Macready AL, Butler LT, Kennedy OB, Ellis JA, Williams CM, Spencer JP. Cognitive tests used in chronic adult human randomised controlled trial micronutrient and phytochemical intervention studies. Nutr Res Rev. 2010;23(2):200-29.

[40] Reitan RM, Wolfson D. The Halstead-Reitan Neuropsycholgical Test Battery: Therapy and clinical interpretation. Tucson, AZ: Neuropsychological Press, 1985.

[41] Panzer VP, Bandinelli S, Hallett M. Biomechanical assessment of quiet standing and changes associated with aging. Arch Phys Med Rehabil. 1995;76(2):151-7.

[42] Amiridis IG1, Hatzitaki V, Arabatzi F. Age-induced modifications of static postural control in humans. Neurosci Lett. 2003;350(3):137-40.

[43] Tinetti ME, Speechley M, Ginter SF. Risk factors for falls among elderly persons living in the community. N Engl J Med. 1988;319:1701-7.

[44] Murphy J, Isaacs B. The post-fall syndrome: A study of 36 elderly patients. Gerontology. 1982;28:265-70.

[45] Arfken CL, Lach HW, Birge SJ, Miller JP. The prevalence and correlates of fear of falling in elderly persons living in the community. Am J Public Health. 1994;84:565-70.

[46] Tinetti ME, Mendes de Leon CF, Doucette JT, Baker DI. Fear of falling and fall-related efficacy in relationship to functioning among community-living elderly persons. J Gerontol. 1994;49:M140-7.

[47] Fernie GR, Gryfe CI, Holliday PJ, Llewellyn A. The relationship of postural sway in standing to the incidence of falls in geriatric subjects. Age Ageing. 198;211:11-6.

[48] Nagano H, Begg RK, Sparrow WA, Taylor S. A comparison of treadmill and overground walking effects on step cycle asymmetry in young and older individuals. J Appl Biomech. 2013;29(2):188-93.

[49] El Haber N, Erbas B, Hill KD, Wark JD. Relationship between age and measures of balance, strength and gait: Linear and non-linear analyses. Clinical Science. 2008;114(12):719-27.

[50] Lajoie Y, Teasdale N, Bard C, Fleury M. Upright standing and gait: Are there changes in attentional requirements related to normal aging? Exp Aging Res. 1996;22(2):185-98.

[51] Ferrandez AM, Pailhous J, Durup M. Slowness in elderly gait. Exp Aging Res. 1990;16(1-2):79-89.

[52] Delbaere K, Sturnieks DL, Crombez G, Lord SR. Concern about falls elicits changes in gait parameters in conditions of postural threat in older people. J Gerontol A Biol Sci Med Sci. 2009;64(2):237-42. 
[53] Chamberlin ME, Fulwider BD, Sanders SL, Medeiros JM. Does fear of falling influence spatial and temporal gait parameters in elderly persons beyond changes associated with normal aging? J Gerontol A Biol Sci Med Sci. 2005;60(9):1163-7.

[54] Morris RGM, Garrud P, Rawlins JNP, O'Keefe J. Place navigation impaired in rats with hippocampal lesions. Nature. 1982;297:681-3.

[55] Devan BD, Goad EH, Petri HL. Dissociation of hippocampal and striatal contributions to spatial navigation in the water maze. Neurobiol Learn Mem. 1996;66(3):305-23.

[56] Jacobs WJ, Laurance HE, Thomas GF. Place learning in virtual space I: Acquisition, overshadowing, and transfer. Learning and Motivation. 1997;28:521-41.

[57] Hamilton DA, Driscoll I, Sutherland RJ. Human place learning in a virtual Morris water task: Some important constraints on the flexibility of place navigation. Behav Brain Res. 2002;129:159-70.

[58] Hamilton DA, Johnson TE, Redhead ES, Verney SR. Control of rodent and human spatial navigation by room and apparatus cues. Behav Processes. 2009;81:154-69.

[59] Astur RS, Taylor LB, Mamelak AN, Philpott L, Sutherland RJ. Humans with hippocampus damage display severe spatial memory impairments in a virtual Morris water task. Behavioural Brain Research. 2002;132:77-84.

[60] Goodrich-Hunsaker NJ, Livingstonem SA, Skelton RW, Hopkins RO. Spatial deficits in a virtual water maze in amnesic participants with hippocampal damage. Hippocampus. 2010;20(4):481-91.

[61] Woolley DG, Laeremans A, Gantois I, Mantini D, Vermaercke B, Op de Beeck HP, Swinnen SP, Wenderoth N, Arckens L, D'Hooge R. Homologous involvement of striatum and prefrontal cortex in rodent and human water maze learning. Proc Natl Acad Sci USA. 2013;110(8):3131-6.

[62] Driscoll I, Hamilton DA., Petropoulos H, Yeo RA, Brooks WM, Baumgartner RN, Sutherland RJ. The aging hippocampus: Cognitive, biochemical and structural findings. Cereb Cortex. 2003;13:1344-51.
[63] Moffat SD, Kennedy KM, Rodrigue KM, Raz N. Extrahippocampal contributions to age differences in human spatial navigation. Cereb Cortex. 2007;17(6):1274-82.

[64] Corrigan JD, Hinkeldey NS. Relationships between parts A and B of the Trail Making Test. J Clin Psychol. 1987;43(4):402-9.

[65] Compton DM, Bachman LD, Brand D, Avet TL. Ageassociated changes in cognitive function in highly educated adults: Emerging myths and realities. Int J Geriatr Psychiatry. 2000;15(1):75-85.

[66] Tombaugh TN. Trail Making Test A and B: Normative data stratified by age and education. Arch Clin Neuropsychol. 2004;19(2):203-14.

[67] Joseph JA, Shukitt-Hale B, Denisova NA, Bielinski D, Martin A, McEwen JJ, Bickford PC. Reversals of age-related declines in neuronal signal transduction, cognitive, and motor behavioral deficits with blueberry, spinach, or strawberry dietary supplementation. J Neurosci. 1999;19(18):8114-21.

[68] Shukitt-Hale B, Miller MG, Chu YF, Lyle BJ, Joseph JA. Coffee, but not caffeine, has positive effects on cognition and psychomotor behavior in aging. Age. 2013;35(6):2183-92.

[69] Shukitt-Hale B, Cheng V, Joseph JA. Effects of blackberries on motor and cognitive function in aged rats. Nutr Neurosci. 2009;12(3):135-40.

[70] Shukitt-Hale B, Kalt W, Carey AN, Vinqvist-Tymchuk M, McDonald J, Joseph JA. Plum juice, but not dried plum powder, is effective in mitigating cognitive deficits in aged rats. Nutrition. 2009;25(5):567-73.

[71] Willis LM, Shukitt-Hale B, Cheng V, Joseph JA. Dosedependent effects of walnuts on motor and cognitive function in aged rats. Br J Nutr. 2009;101(8):1140-4.

[72] Shukitt-Hale B, Carey A, Simon L, Mark DA, Joseph JA. Effects of Concord grape juice on cognitive and motor deficits in aging. Nutrition. 2006;22(3):295-302.

[73] Devore EE, Kang JH, Breteler MM, Grodstein F. Dietary intakes of berries and flavonoids in relation to cognitive decline. Ann Neurol. 2012;72(1):135-43. 